\title{
Novel Design of a Biaxial and Four-Wheeled Robot Capable of Steering
}

\author{
Guiping Lan ${ }^{1}$, Yujun Wang ${ }^{1}$, Can Fang ${ }^{1}$, Min Yi ${ }^{1}$ \\ ${ }^{1}$ College of Computer and Information Science, Southwest University, BeiBei 400415, China
}

\begin{abstract}
The paper presents a biaxial and four-wheeled robot, mainly composed of two axle, two circular wheels and two three-leaved wheels. By analyzing the difference between the velocity of the circular wheel and three-leaved wheel in same axle, the steering principle of the difference velocity is proved. The three-leaved wheels are installed by a complementary phase method to ensure the stability of the robot walking. Through the steering principle, the control method of robot' $\mathrm{s}$ forward, backward, turn left and turn right is designed. A large number of experimental results show that the robot has the characteristics of high steering efficiency, simple mechanical structure and easy to control.
\end{abstract}

\section{Introduction}

In order to the wheeled robot has better mobile efficiency, many scholars have designed various structures for steering. Fu Yi-li, et al [1] proposed a variety of steering methods using omni-directional wheeled mode. Chang jian,et al [2] proposed a shape-shifting robot skid-turning with line configuration method. Gao Jun-xiao et al [3] proposed GA fuzzy neural network algorithm to achieve the steering control of the robot. Yan Yong-bao et al [4] proposed a independent drive skid-steering vehicle. $\mathrm{Li}$ Xue-yuan et al [5] proposed a steering method with difference velocity. Xing Jie [6] analyzed differential steering and torque matching of 4-WD mobile robot. Ye Chang-long et al [7] designed an omnidirectional mobile robot with MY wheels. Qian J et al [8] designed and developed an omni-directional mobile robot oriented to an intelligent manufacturing system. $\mathrm{Zi}$ B et al [9]designed a winding hybrid-driven cable parallel manipulator. They proposed the mechanical structures or algorithms are too complex and difficult to control.

To cope with these problems, the biaxial and fourwheeled robot is proposed in this paper that was focused on small-size, high efficiency of movement, simple mechanical structure, easy to control and strong capability of steering. This paper is organized as follows: Section 2 describes the mechanical structure of the robot. Section 3 detail the steering principle. The experimental results are shown in Section 5. Finally, Section 6 is the conclusions.

\section{Conception of the robot}

\subsection{Mechanical structure}

The paper presents a biaxial and four-wheeled robot, composed of two axles, two transmission mechanisms, two motors, two circular wheels and two three-leaved wheels, as shown in Figure 1. Each end of the axle is mounted with a circular wheel and a three-leaved wheel, respectively. Two circular wheels and two three-leaved wheels are installed on both sides of the body. When each motor drives the axle through the transmission mechanism, the circular wheel and the three-leaved wheel are rotated synchronously. The transmission ratio of the transmission mechanism is $1: 1$. The phase difference between two three-leaved wheels is $60^{\circ}$. The radius of the circular wheel and the three-leaved wheel is same. The angle between each leaf of the three -leaved wheel is $60^{\circ}$ as shown in Figure 2. Figure 3 is a physical diagram of a biaxial four-wheeled robot.

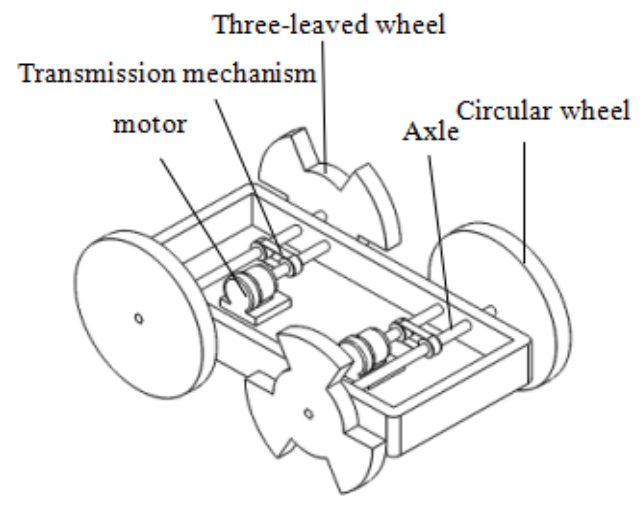

Figure 1. 3D model of the robot 


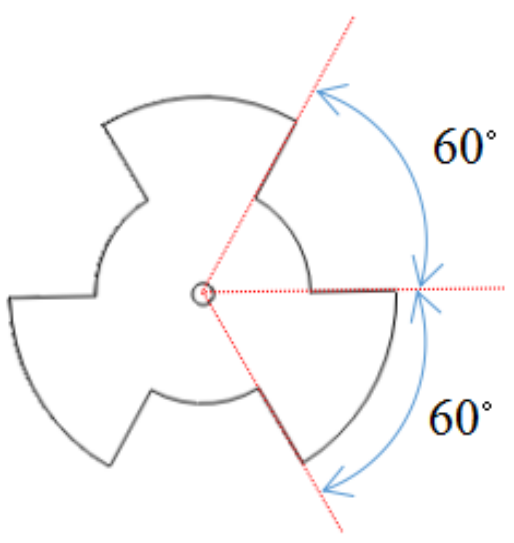

Figure 2. Three-leaved wheel structure

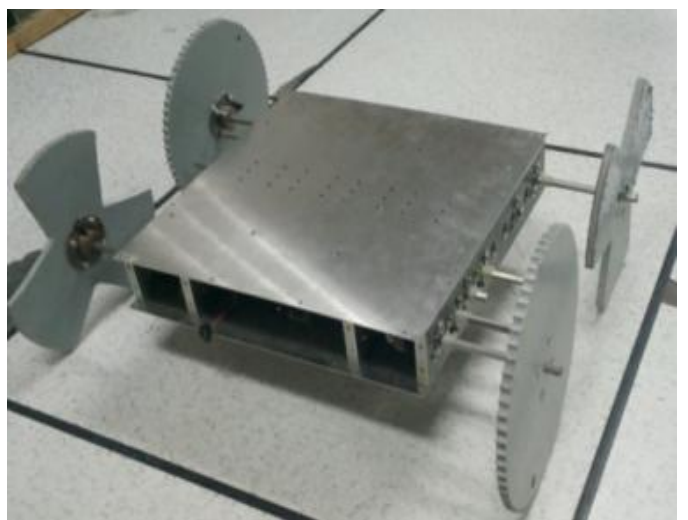

Figure 3. The biaxial and four-wheeled robot

\subsection{Control system}

In order to achieve high motion efficiency, the design of the control system is essential. Two low-torque motors are used to drive the wheels with encoder. Two motor driving modules can be used to drive the motors. The microcontroller (ATMega 328p) is used to control the motor driving modules. And the MCU receives control commands from the host computer. The block diagram of the robot control system is shown in Figure 4.

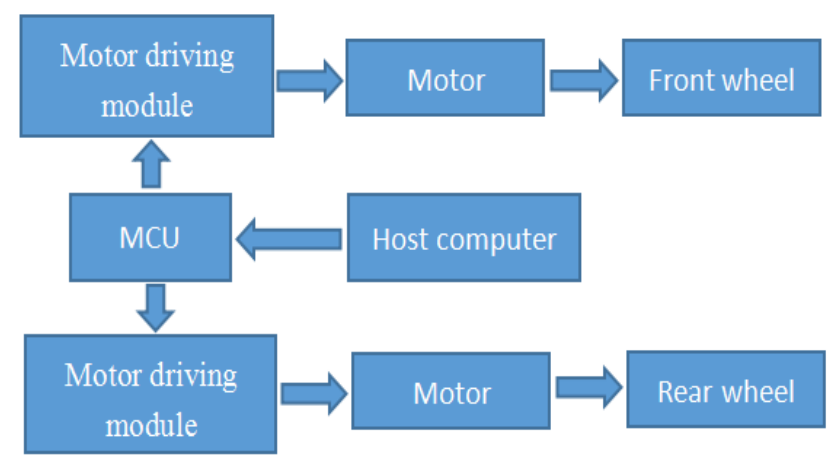

Figure 4. Robot control system

\section{Motion analysis}

In structured environment, wheeled robots have four main forms of movement, forward, backward, turn left and turn right. The circular wheel and the three-leaved wheel on the same axle have the same angular velocity. Assuming that the time required for the wheels to rotate one round is $\mathrm{t}$, the walking distance of the circular wheel and three-leaved wheel on the ground can be obtained by :

$$
\begin{aligned}
& S_{1}=R \omega t \\
& S_{2}=\frac{1}{2} R \omega t
\end{aligned}
$$

Where $S_{1}, S_{2}$ are the walking distance of the circular wheel and three-leaved wheel on the ground, respectively; $R$ is the radius of the circular wheel and three-leaved wheel. $\omega$ is the angular velocity of the circular wheel and three-leaved wheel.

The walking velocity of the circular wheel and threeleaved wheel on the ground can be obtained by :

$$
\begin{aligned}
& v_{1}=\frac{d S_{1}}{d t}=R \omega \\
& v_{2}=\frac{d S_{2}}{d t}=\frac{1}{2} R \omega
\end{aligned}
$$

Since the speed of the circular wheel is greater than the speed of the three-leaved wheel, the robot rotates to the side of the three-leaved wheel, as shown in Figure 5. When the motor drives the circular wheel and threeleaved wheel forward, a clockwise torque $\left(\tau_{1}\right)$ is generated, as shown in Figure 5 (a). When the motor drives the circular wheel and three-leaved wheel backward, a counterclockwise torque $\left(\tau_{2}\right)$ is generated, as shown in Figure 5 (b).

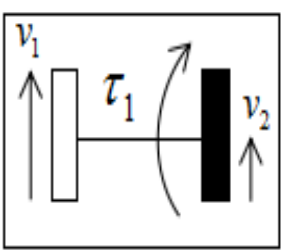

(a)

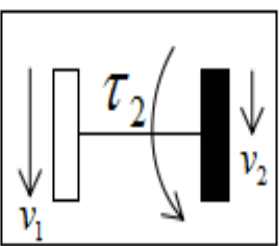

(b)
Figure 5. Coaxial steering principle

(White box is circular wheel, black box is three-leaved wheel)

From the steering principle mentioned above, we can get four kinds of biaxial four-wheeled robot movement: forward, backward, turn left and turn right. Assuming that the output power of the two motors is the same, the friction coefficient between the wheels and the ground is the same.

When the robot moves forward (or backward), the front and rear motors simultaneously drive the circular wheels and three-leaved wheels forward (or backward) as shown in Figure 6(a)(b). In the process of movement the following relationship: 


$$
\begin{aligned}
& v_{1}=v_{4} \\
& v_{2}=v_{3} \\
& \tau_{f}=\tau_{r}
\end{aligned}
$$

Where $v_{1}, v_{4}$ are the walking velocity of the front circular wheel and the rear circular wheel on the ground, $v_{1}, v_{4}$ have the same value and direction; $v_{2}, v_{3}$ are the walking velocity of the front three-leaved wheel and the rear three-leaved wheel on the ground. $v_{2}, v_{3}$ have the same value and direction; $\tau_{f}, \tau_{r}$ are the torque of the front axle and the rear axle on the ground. $\tau_{f}, \tau_{r}$ have the same value and the opposite direction.

When the robot turns left, the front motor drives the circular wheel and three-leaved wheel backward and the rear motor drives the circular wheel and three-leaved wheel forward as shown in Figure 6(c). In the process of movement the following relationship:

$$
\begin{aligned}
& v_{1}=v_{4} \\
& v_{2}=v_{3} \\
& \tau_{f}=\tau_{r}
\end{aligned}
$$

Where $v_{1}, v_{4}$ have the same value and the opposite direction; $v_{2}, v_{3}$ have the same value and the opposite direction; $\tau_{f}, \tau_{r}$ have the same value and the same direction.

When the robot turns right, the front motor drives the circular wheel and three-leaved wheel forward and the rear motor drives the circular wheel and three-leaved wheel backward as shown in Figure 6(d). In the process of movement the following relationship:

$$
\begin{aligned}
& v_{1}=v_{4} \\
& v_{2}=v_{3} \\
& \tau_{f}=\tau_{r}
\end{aligned}
$$

Where $v_{1}, v_{4}$ have the same value and the opposite direction; $v_{2}, v_{3}$ have the same value and the opposite direction; $\tau_{f}, \tau_{r}$ have the same value and the same direction.

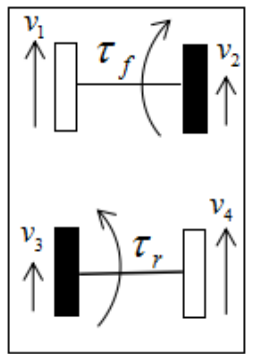

(a)

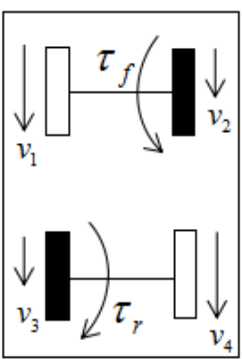

(b)

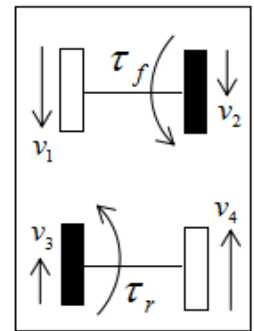

(c)

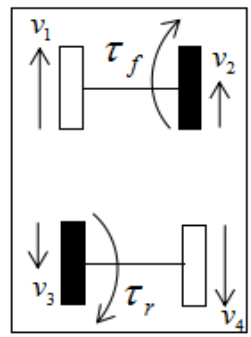

(d)
Figure 6. The four movements of the robot

\section{Experiment}

In order to verify the steering principle of the robot and test the efficiency of walking, a series of experiments were carried out. In the experiment, four batteries(MCOBEAM / 3400Mah / 3.7V ) are used the power of the DSAWbot. MCU is Arduino nano V3.0. The motor drive module uses TSK-BTN7971.Two motors(Germany Faulhaber23420L012CR) can be used to drive the wheels. The size of robot used in this experiment was $310 \mathrm{~mm}$ by $310 \mathrm{~mm}$ by $200 \mathrm{~mm}$ for the length, width and high, respectively. The radius of wheel is $80 \mathrm{~mm}$. Figure 6 is the process of the left turn of the robot. Figure 7 is the process of the right turn of the robot. Experiments show that the steering principle proposed in this paper is correct and can achieve the left and right turn of the robot. Experiment result show that the robot can to steer on the ground. In the case of limited power and size, the walking efficiency of the robot are maximized. The relevant experimental results are shown in Table 1.A large number of experimental results show that the robot has the characteristics of high steering efficiency, simple mechanical structure and easy to control as shown in Figure 7 and Figure 8.

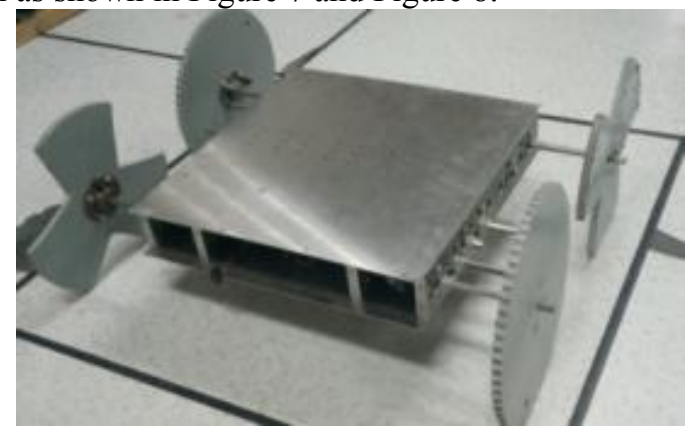

(a)

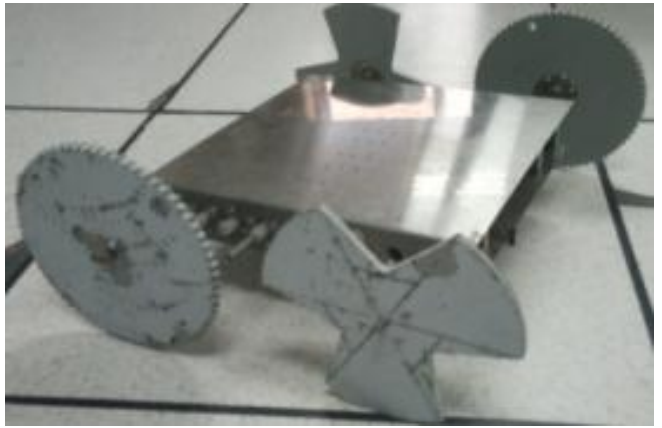

(b) 


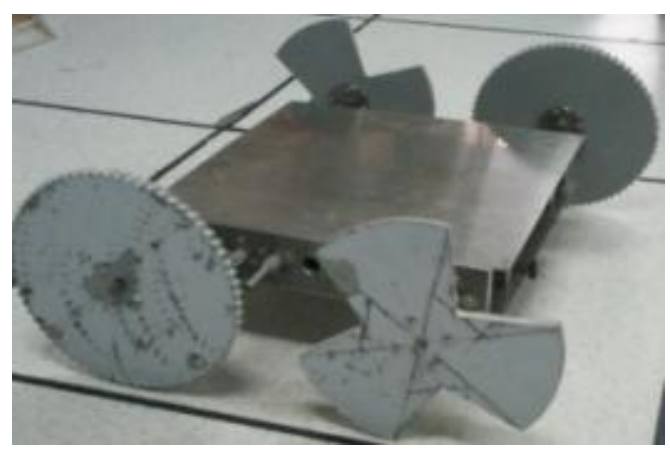

(c)

Figure 7. Turn left

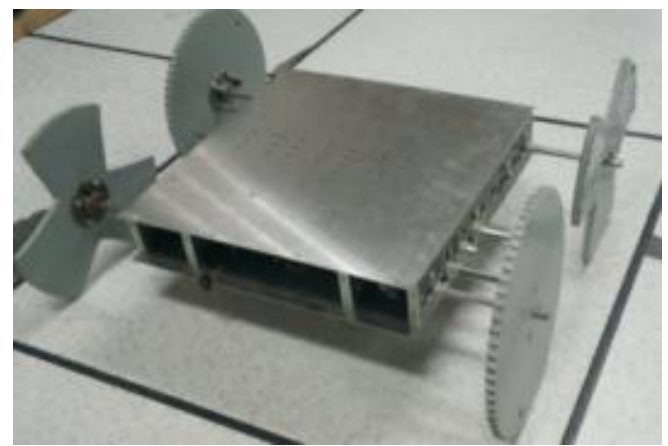

(a)

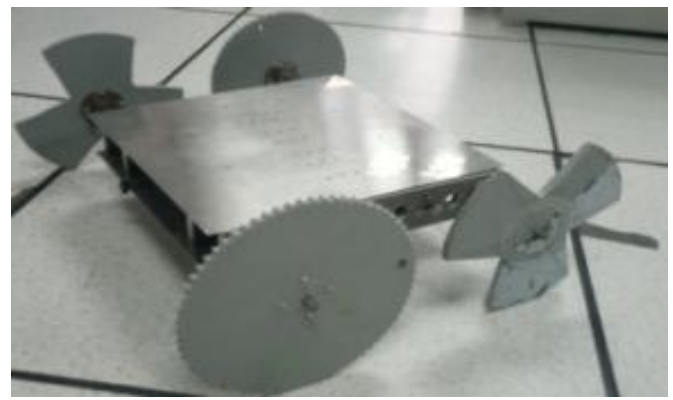

(b)

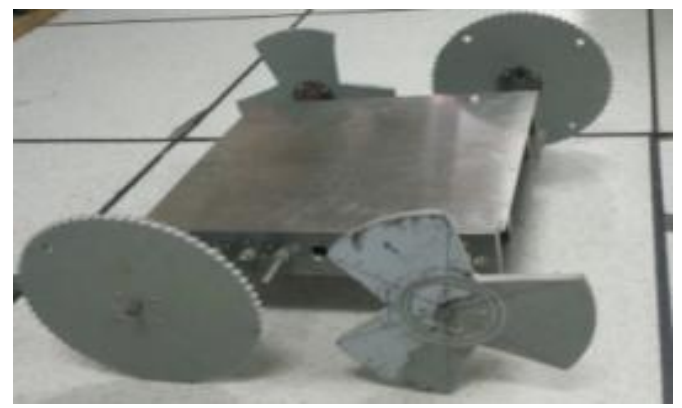

(c)

Figure 8. Turn right

Table 1. Specifications of the robot

\begin{tabular}{|l|l|}
\hline Parameters & robot \\
\hline Total weight & $8.47 \mathrm{~kg}$ \\
\hline load capacity & $4.25 \mathrm{~kg}$ \\
\hline $\begin{array}{l}\text { Speed in structure } \\
\text { environment }\end{array}$ & $3.16 \mathrm{~km} / \mathrm{h}$ \\
\hline Size & $\begin{array}{l}310 \mathrm{~mm} * 310 \mathrm{~mm} * 20 \\
0 \mathrm{~mm}\end{array}$ \\
\hline Radius & $80 \mathrm{~mm}$ \\
\hline
\end{tabular}

\section{Conclusion}

In this paper, a biaxial four-wheeled robot is proposed, which utilizes the phase complementarity between two three-leaved wheels. Each time there are two circular wheels and one three leaved wheel on the ground, which guarantees the stability of the robot during the walking process. By analyzing the difference between the velocity of the coaxial circular wheel and the three-leaved wheel during the movement, the four kinds of motion principle of the wheeled robot are proved, and the principle is further verified by experiment.

(1) Each axle of the three-leaved and circular wheel have the same angular velocity, the robot walking more efficient.

(2) Every moment, the robot has three wheels on the ground, to ensure the stability of walking.

(3) The steering principle of coaxial difference velocity can be used to achieve robot's movement.

(4) The mechanical structure of the biaxial four-wheeled robot is simpler.

In the complex environment, the robot can work autonomically and stably. The robot is highly efficient either as a post-disaster detection and rescue robot or as a service robot.

\section{Acknowledgments}

This work is financially supported by "Fundamental Research Funds for the Central Universities"(XDJK2017D070, XDJK2017D063).

\section{References}

1. Fu Yi-li, Li Han, Xu He. Research on several kinds of steering mode of OWMR[J]. Manufacturing Automation, 2005, 27(10): 33-37.

2. Chang Jian, Wu Cheng-dong, Li Bin. Shape-shifting robot skid-turning with line configuration $[\mathrm{J}]$. Journal of Mechanical Engineering, 2012, 48(7): 915.

3. Gao Jun-yao, Lu Ji-lian. Steering control of wheeled mobile robot using GA fuzzy neural network[J]. Transactions of Beijing Institute of Technology, 2003, 23(2): 176-180.

4. Yan Yong-bao, Zhang Yu-nan, Yan Nanming. Simulation study of motion control algorithm for a six-wheel independent drive skid-steering vehicle[J]. Acta Armamentarii, 2013, 34(11):14611468.

5. Li Xue-yuan, Zhang Yu, Hu Ji-bin. Skid-steering resistance characteristics of wheeled vehicle[J], Acta Armamentarii. 2013, 32(12): 1433-1438.

6. Xing Jie. Differential steering and torque matching of 4-WD mobile robot[D]. Shanghai: Tongji University, 2008.

7. Ye Chang-long, Li Huai-yong, Ma Shu-gen. Kinematics study on an omnidirectional mobile robot with MY wheels[J]. robot, 2012, 34(2): 144151. 
8. Qian J, Zi B, Wang D, et al. The Design and Development of an Omni-Directional Mobile Robot Oriented to an Intelligent Manufacturing System. Sensors, 2017, 17(9): 2073; doi:10.3390/s17092073

9. Zi B, Sun H, Zhang D. Design, analysis and control of a winding hybrid-driven cable parallel manipulator. Robotics and Computer-Integrated Manufacturing, 2017, 48: 196-208. 